\title{
Borehole Effects on Coaxial and Coplanar Logs within Laminated Formations with Anisotropic Shale
}

\author{
Paulo Roberto de Carvalho ${ }^{1}$, Cícero Régis ${ }^{2,3}$, and Valdelírio da Silva e Silva ${ }^{2}$, \\ ${ }^{1}$ Universidade Federal Rural da Amazônia, ${ }^{2}$ Universidade Federal do Pará, ${ }^{3}$ INCT de Geofísica do Petróleo.
}

Copyright 2021, SBGf - Sociedade Brasileira de Geofísica

This paper was prepared for presentation during the $17^{\text {th }}$ International Congress of the Brazilian Geophysical Society, held in Rio de Janeiro, Brazil, 8-11 November 2021.

Contents of this paper were reviewed by the Technical Committee of the $17^{\text {th }}$ International Congress of The Brazilian Geophysical Society and do not necessarily represent any position of the SBGf, its officers or members. Electronic reproduction or storage of any part of this paper for commercial purposes without the written consent of The Brazilian Geophysical Society is prohibited.

\section{Abstract}

This paper performs an analysis of the borehole effects in coaxial and coplanar electromagnetic logs within laminated formation models with isotropic and anisotropic shale laminae. This study compares results from a 3D Vector Finite Element program (with borehole) and a 1D-Analytic code (no borehole). The results reproduce some wellknown characteristics of these logs. In comparison with the coaxial configuration, the coplanar logs show a stronger horning effect in front of the bed boundaries; a stronger skin effect to the conductivity media; and a more prominent oscillation in the laminated formation. In addition, feature changes (curves with angular or smooth shapes) occur on the coaxial and coplanar responses as the dipping angle varies. We conclude that the coplanar responses are more sensitive to the anisotropy and the borehole effects than the coaxial responses although they have opposite behaviors with respect to the dipping angle, i.e., for small angles where the coaxial is least sensitive to these effects, the coplanar is most sensitive, and for large dip angles where the coaxial is most sensitive, the coplanar is least sensitive.

\section{Introduction}

The deepwater turbidite reservoirs from Campos Basin comprise one of the most important petroleum accumulations offshore Brazil. These reservoirs can be very complex and heterogeneous, ranging from massive thick sands to highly laminated sand/shale sequences. A laminated reservoir consists of thin layers alternating between conductive water-bearing shales and resistive oil-bearing sands (Gomes et al., 2002), as illustrated in Figure 1.

Thinly laminated reservoirs are described by a typical effective electrical anisotropy because the single layers cannot be individually resolved by the resistivity induction tools. The horizontal conductivity, $\sigma_{h}$, parallel to the layers is more strongly controlled by the shale, while the vertical conductivity, $\sigma_{v}$, perpendicular to the layers, is dominated by the resistive hydrocarbon sand zones. This type of anisotropy is usually referred to as structural or macroscopic anisotropy.

From the beginning of this century, the need for more information from Interbedded sand-shale turbidite sequences prompted the development of the triaxial or multicompo-

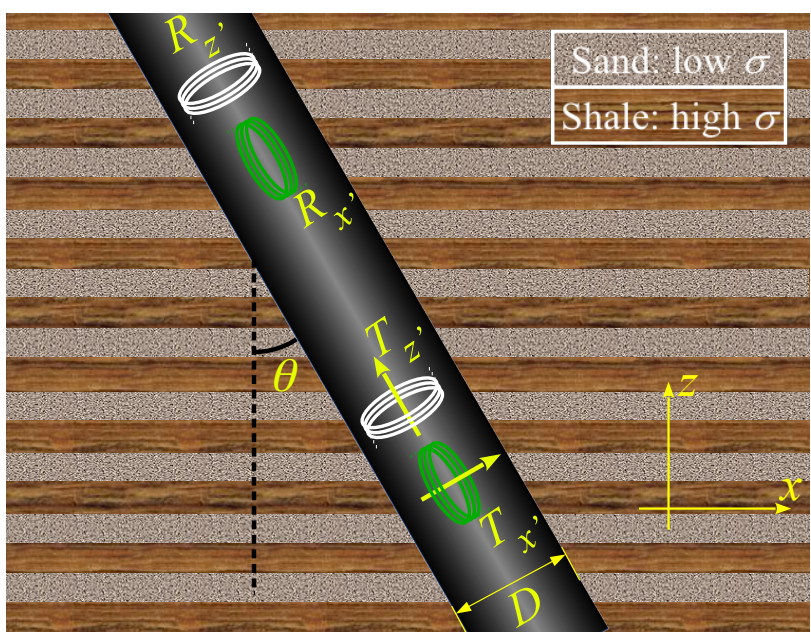

Figure 1: Laminated sand/shale sequence and illustration of the transmitters and receivers of the coaxial and coplanar coil configurations.

nent wireline induction tool, with nine coupling components, that have been successfully used to determine formation anisotropy and bedding dip angle (Kriegshäuser et al., 2000). Currently, besides being the main location tool of finely laminated reservoirs, triaxial sources and sensors are also applied in many situations of asymmetric geometry, such as locating dissolution cavities (vugs) and fractured zones in the vicinity of the wells, monitoring invasion fronts in horizontal wells, among others.

The simplest multicomponent triaxial induction tool consists of three mutually orthogonal coil transmitters and receivers. Figure 1 illustrates two of the nine possible transmitter-receiver combinations: the coaxial configuration, where the dipole moment of the source and the receiver are aligned with the borehole axis, and a coplanar configuration with the dipole moment of the source normal to the axis in the $(x, z)$ plane. The borehole diameter is $D$.

Transversely isotropic ( $\mathrm{TI})$ homogeneous media have the same resistivity in every direction in the bedding plane, but a different resistivity normal to it. TI anisotropy is a reasonable assumption based on normal depositional processes. For TI media with a vertical axis of symmetry (TIV), Kaufman and Ytskovich (2017) show that, in the low frequency range, the anisotropy ratio $\lambda^{2}$ is

$$
\lambda^{2}=\frac{\sigma_{h}}{\sigma_{v}} \approx \frac{\operatorname{Im}\left\{H_{z z}\right\} / H_{z z}^{(0)}}{\operatorname{Im}\left\{H_{x x}\right\} / H_{x x}^{(0)}}, \quad \omega \rightarrow 0, \quad \text { or } \quad \frac{L}{\delta} \ll 1,
$$

where $\sigma_{h}$ and $\sigma_{v}$ are the horizontal and vertical conductiv- 
ities, $L$ is the transmitter-receiver offset, $H_{z z}^{(0)}=m_{z} /\left(2 \pi L^{3}\right)$ and $H_{x x}^{(0)}=m_{x} /\left(4 \pi L^{3}\right)$ are the coaxial and coplanar direct mutual coil coupling, respectively, and $\delta$ is the plane wave skin depth. Anderson et al. (2008) show that this anisotropy index is a useful measurement because it alerts the log analyst to look for potential laminated pay-reservoir.

Complex apparent conductivities may be calculated from the coaxial $\sigma_{a}^{c x}$ and coplanar $\sigma_{a}^{c p}$ coil configurations, assuming an infinite homogeneous isotropic medium (Zhang et al., 2012):

$$
\begin{aligned}
\sigma_{a}^{c x} & =\sigma_{R}^{c x}+i \sigma_{X}^{c x}=i \frac{4 \pi L}{\omega \mu} H_{z z}, \\
\sigma_{a}^{c p} & =\sigma_{R}^{c p}+i \sigma_{X}^{c p}=i \frac{8 \pi L}{\omega \mu} H_{x x} .
\end{aligned}
$$

The real parts $\sigma_{R}^{c x}$ and $\sigma_{R}^{c p}$ are named quadrature or resistive components and the imaginary parts $\sigma_{X}^{c x}$ and $\sigma_{X}^{c p}$ are the inphase or reactive components.

The coaxial and coplanar mutual coupling signals $\left(H_{z z}^{(o)}, H_{x x}^{(o)}\right)$ are part of the imaginary parts of the conductivities and are several orders of magnitude greater than the formation signals. Actual field tools usually contain additional "bucking" coils to cancel these large mutual coupling signals. However, since it is straightforward to calculate analytically and remove them computationally (Anderson et al. 2002) we do not model bucking coils. The imaginary parts of the apparent conductivity without direct coupling are denoted by $\sigma_{X F}^{c x}$ and $\sigma_{X F}^{c p}$.

The effective horizontal $\left(\sigma_{h}\right)$ and vertical $\left(\sigma_{v}\right)$ conductivities of a sand-shale $\left(\sigma_{s d}\right.$ and $\left.\sigma_{s h}\right)$ thinly laminated formation, when their laminae thicknesses are less than the tool's vertical resolution, are obtained by weighted arithmetic and harmonic means, respectively:

$$
\begin{aligned}
& \sigma_{h}=\sigma_{s d} V_{s d}+\sigma_{s h} V_{s h}, \\
& \sigma_{v}=\left(\frac{V_{s d}}{\sigma_{s d}}+\frac{V_{s h}}{\sigma_{s h}}\right)^{-1},
\end{aligned}
$$

where the weights $V_{s d}$ and $V_{s h}=1-V_{s d}$ are the volume fractions of each material, that can be obtained, for example, by spectroscopy probe (Anderson et al., 2008).

Equation 4 explains the strong dependence of $\sigma_{h}$ to the water-bearing shale laminae (high conductivity) and the poor sensitivity to the oil-bearing sand laminae (low conductivity).

Thus, sand laminae conductivity can be estimated from the horizontal (coaxial signal) and vertical (coplanar signal) apparent conductivities and applied in the classical Waxman and Smits (1968) model to estimate the water saturation in oil-bearing shaly sand reservoirs.

Originally, this method (Eqs. 4 and 5) assumed sand and shale laminae were isotropic. Lab measurements and field test results of the triaxial induction tool, however, show that shale formations often have anisotropic conductivity $\left(\lambda^{2}=1\right.$ to 8), originating from their micro-bedding structure with dimensions bellow measurement resolution. Thus, in shales this type of anisotropy is a function of the compaction (clay porosity). This is usually referred to as intrinsic or microscopic anisotropy, and it is usually weaker than the structural anisotropy of laminated formations. Clavaud et al. (2005) show that intrinsic shale anisotropy plays an important role in the estimation of conductivities in finely laminated sand-shale reservoirs.

According to Moinfar et al. (2010) the borehole and the invasion zone effects on multi-component induction measurements can be significant, mainly in water-base muds. These effects may give rise to electrical pseudo-anisotropy in isotropic reservoirs. Therefore, a quantitative assessment of the influence of the borehole on well logging data may contribute with important information to help in interpretation.

To study the effect of the borehole we have implemented a 3D Vector Finite Element program to simulate electromagnetic well logs in anisotropic formations. Using this program, we simulate well log data from vertical, as well as inclined wells using both coaxial and coplanar coil configurations.

Here we present the results of a comparative analysis of the Tl-anisotropy level on the coaxial and coplanar responses in one-dimensional (no borehole) and threedimensional laminated sand-shale models, with isotropic and anisotropic laminae, traversed by a borehole filled with a conductive water-based mud.

\section{Theory and method}

The layered 1D problem is formulated using the mathematical tools described by Kaufman and Ytskovich (2017), generalized to multi-layered TIV media. The solutions are written as integrals of the Hankel transform, which are evaluated numerically using the Quadrature With Extrapolation (QWE) method as presented by Key (2012). The basic difference between the solutions that we have implemented (Carvalho et al., 2010) for the 1D isotropic and $1 \mathrm{D}$ anisotropic problems is the manner of recursively computing the transmission and reflection coefficients on the interfaces. For the isotropic case, explicit analytic expressions are written for these coefficients using recurrence relations, whereas in the anisotropic problem all coefficients are determined by the solution of a system of linear equations whose size is proportional to the number of layers in the model.

The 3D problem is solved with an implementation of the Vector Finite Element method, using a secondary magnetic field formulation and following the steps presented by Jin (2015). The dipole sources are in an infinite homogeneous isotropic space with the same conductivity as the drilling mud that simulates the environment inside the borehole. This choice for the primary medium means that the secondary media occupy all the space outside the borehole, so that the primary electric field from the sources needs to be calculated in a great number of points in the mesh. However it presents two important advantages: it will easily allow the simulation of different geometries for the borehole in future work and it results in the primary electric field (Ward and Hohmann, 1987, p. 176) being calculated using an extremely simple formula, with minimum computational effort.

The problem is formulated to solve directly for the secondary magnetic field, which obviates the need to calculate numerical derivatives.

The system of linear equations generated by the Vector 
Finite Element method needs to be solved twice (once for each dipole) for every tool position in the profile, with the same complex sparse coefficient matrix. We chose to use a direct parallel solver, because a direct approach allows the factoring of the coefficient matrix only once, solving the multiple systems every time with only a phase of forward and backward susbtitutions. This means that the problem is highly demanding in terms of computer memory, but can be solved in shorter times than with an iterative solution.

The profiles presented here will be in straight boreholes positioned in the $(x, z)$ plane, and only the coaxial and coplanar configurations will be simulated. Using the coordinate system illustrated in Figure 1, in both 1D and 3D cases the observed fields for the coaxial $\left(H_{z^{\prime} z^{\prime}}\right)$ and coplanar $\left(H_{x^{\prime} x^{\prime}}\right)$ coil configurations with source dipole moments $m_{x^{\prime}}=$ $m_{z^{\prime}}=m$ are the combination of four signals: the vertical $\left(H_{z z}\right.$ and $\left.H_{x z}\right)$ and horizontal $\left(H_{z x}\right.$ and $\left.H_{x x}\right)$ components from the vertical (VMD) and horizontal (HMD) dipole sources. These are the ones needed to simulate the responses at any dip angle $\theta$ measured in relation to the vertical $z$-axis:

$H_{z^{\prime} z^{\prime}}=m\left[H_{x x} \sin ^{2} \theta+\left(H_{x z}+H_{z x}\right) \sin \theta \cos \theta+H_{z z} \cos ^{2} \theta\right]$
$H_{x^{\prime} x^{\prime}}=m\left[H_{x x} \cos ^{2} \theta-\left(H_{x z}+H_{z x}\right) \sin \theta \cos \theta+H_{z z} \sin ^{2} \theta\right]$

The sensitivity of the coaxial $\left(H_{z^{\prime} z^{\prime}}\right)$ and coplanar $\left(H_{x^{\prime} x^{\prime}}\right)$ signals to anisotropy is complementary: for small dip angles where the coaxial is least sensitive to anisotropy, the coplanar is most sensitive, and for large dip angles where the coaxial is most sensitive, the coplanar is least sensitive.

\section{Results}

In our simulations, the tool transmitters are magnetic point dipoles operating at $20 \mathrm{kHz}$ and the source-receiver offset is $L=1.0 \mathrm{~m}$. The borehole diameter is represented by $D$ and the dip angle is $\theta$.

According to Anderson et al. (1992) a laminated anisotropy level close to $\lambda^{2}=2$ is a typical contrast for actual logging. Thus, in Figures 3 to 4, we have modeled a laminated formation containing resistive sands $\left(\sigma_{s d}=0.2 \mathrm{~S} / \mathrm{m}\right)$ alternating with conductive isotropic shales $\left(\sigma_{s h}=1.0 \mathrm{~S} / \mathrm{m}\right)$. The sand-shale laminae thicknesses are equal $\left(V_{s d}=V_{s h}=\right.$ $50 \%$ ) and value $0.5 \mathrm{~m}$. Thus, applying equations 4 and 5 the equivalent horizontal and vertical conductivities and anisotropy ratio from an equivalent anisotropic bed are

$$
\sigma_{h}=0.6 \mathrm{~S} / \mathrm{m}, \quad \sigma_{v}=\frac{1}{3} \mathrm{~S} / \mathrm{m}, \quad \lambda_{b}^{2}=\frac{\sigma_{h}}{\sigma_{v}}=1.8 .
$$

Figure 2 shows a validation exercise with a comparison of the $3 D$ and $1 D$ logs for the laminated formation with the values as in Equation 8 ) in a dipping $\left(60^{\circ}\right)$ well. In this case, to simulate the measurements in the 3D code, the borehole diameter is assumed exceedingly small $(6 \mathrm{~cm}$ diameter) and the mud conductivity equal to the geometric mean of the two laminae conductivities: $\sigma_{m u d}=\sqrt{\sigma_{s h} \sigma_{s d}}=1 / \sqrt{5}$ $\mathrm{S} / \mathrm{m}$. In these conditions, it is expected that the borehole effects are small and the 1D and 3D solutions are similar.

The results show an excellent agreement between the 1D and $3 \mathrm{D}$ solutions within the laminated formation, except for a slight departure from the curves below and above the laminated formation where the conductivity contrast between the mud and formation is greatest. This small effect appears on the resistive logs, mainly on the coplanar signal, because of its well-known (Anderson et al., 2002) strongest skin effect (signal level attenuation and phase shift caused by the mud conductivity).

The resistive coplanar log is also more sensitive to the conductivity variations in the laminae and show a more prominent oscillation than the coaxial log. In addition, the expected coplanar "horns" are observed in both 1D and $3 D$ results. These horns have been shown by Régis et al. (2020) to be associated with the discontinuous current density field parallel to the interfaces between layers, rather than with surface charge build-up from the continuous current across the interface, as was universally accepted since the early 1990s. They are unavoidable features of the coplanar profiles and are slightly smoothed by the influence of the well, a difference hard to notice for this thin well, but more pronounced in regular wider wells, as shown in the next example.

This result indicates a good validation between $1 \mathrm{D}$ and $3 \mathrm{D}$ responses, i.e., less than $1 \%$ difference within the laminated formation, that gives us confidence in the accuracy for more complicated geometries.

Figure 3 shows the $3 \mathrm{D}$ and 1D results for the same laminated formation as in the validation example, but now traversed vertically by a borehole with a $20 \mathrm{~cm}$ diameter, filled with a water based $3 \mathrm{~S} / \mathrm{m}$ mud and without invasion zones. The goal here is to evaluate the percentage difference of the borehole effect on the 3D coaxial and coplanar signals in relation to those of the no-borehole 1D responses. For this, we calculate the Root Mean Square (RMS) in the middle of the laminated formation, from -2.0 $\mathrm{m}$ to $2.0 \mathrm{~m}$, i.e., away from the boundaries to the adjacent infinite half-spaces above and below.

Firstly, notice within the laminated formation a feature change on these resistive vertical responses (angular to smooth shape) due to the cancellation or disappearance of the horns in the laminae interfaces. In addition, there is a curve reversal with respect to the model for both coaxial and coplanar resistive logs which seems to arise from purely geometrical effects of the relative positions of the transmitter and the receiver within the laminae (Carvalho et al., 2018).

Comparing with an equivalent anisotropic bed with conductivities calculated using Eqs. 4 and $5\left(\sigma_{h}=0.6 \mathrm{~S} / \mathrm{m}\right.$, $\sigma_{v}=1 / 3 \mathrm{~S} / \mathrm{m}$ ), note that within the laminated formation the resistive coaxial signal oscillates close to $\sigma_{h}$ whereas the resistive coplanar signal oscillates near $\sigma_{v}$.

Again, the borehole effect is much more pronounced on the resistive signals than on the deeper reactive signals for both coil arrays although this effect is stronger in the coaxial reactive signal $(7.6 \%)$ than on the coplanar reactive signal $(0.7 \%)$. As for the resistive signals, the borehole effect has the opposite behavior, i.e., the coaxial resistive signal increases $(6.9 \%)$ whereas the coplanar resistive signal decreases strongly (25.4\%) due to the accentuated skin effect in this conductive mud.

Figure 4 shows the coaxial and coplanar deviated logs at $60^{\circ}$ in the same laminated model of the Figure 3 . This dipping angle increases the borehole effect on the coaxial reactive $(7.6 \%$ to $8.5 \%)$ and resistive $(6.9 \%$ to $8.7 \%)$ 
signals, as compared to the vertical logs shown in Figure 3. However, reductions are observed on the coplanar reactive $(0.7 \%$ to $0.02 \%)$ and resistive (25.4\% to $15.6 \%)$ signals.

Figure 5 shows the 1D and 3D coaxial and coplanar responses for a laminated formation analogous to the previous examples, but now the model has anisotropic shale $\left(\lambda_{s h}^{2}=2\right)$ with horizontal and vertical conductivities $\sigma_{h}^{s h}=1.0 \mathrm{~S} / \mathrm{m}$ and $\sigma_{v}^{s h}=0.5 \mathrm{~S} / \mathrm{m}$. Thus, there is no vertical conductivity contrast in this formation anisotropic model.

According to Clavaud et al. (2005) replacing $\sigma_{h}^{s h}$ for $\sigma^{s h}$ in Equation 4 and $\sigma_{v}^{s h}$ for $\sigma^{s h}$ in Equation 5 we obtain the anisotropic ratio $\left(\lambda_{b}^{2}=2.1\right)$ from the equivalent anisotropic bed to this laminated formation with anisotropic shale laminae.

Firstly, notice within the laminated formation a new angular feature of the coaxial resistive responses due to the anisotropic shale laminae, whereas the coplanar responses have the same angular shape as seen in Figure 3. The differences between 3D and 1D data due to the borehole effect on the coaxial reactive and resistive logs are $9.9 \%$ and $9.3 \%$, respectively, while on the coplanar reactive and resistive logs are $0.02 \%$ and $15.4 \%$, respectively.

The sensitivity of the coaxial and coplanar responses to the anisotropy and the borehole is opposite, i.e., for small dip angles where the coaxial is least sensitive to these effects, the coplanar is most sensitive, and for large dip angles where the coaxial is most sensitive, the coplanar is least sensitive. The main physical cause of these coaxial and coplanar opposite behaviors is the same: the weight of the horizontal magnetic component of the horizontal dipole $\left(H_{x x}\right)$ contribution on the coaxial and coplanar dipping logs, since this is the only one of the four magnetic field component that has anisotropy sensitivity and strongest skin effect in the Equations 6 and 7.

\section{Conclusion}

The comparative study presented here investigates the influence of the borehole environment in the responses of coplanar and coaxial electromagnetic well logs in laminated formations, using 1D analytical solutions to simulate the logs without the borehole and a 3D vector finite element program to model the responses in the same formations with the presence of a borehole.

The results from the show that the coplanar responses are more sensitive to the anisotropy and the borehole effects than the coaxial responses although they have opposite behaviors with respect to the dipping angle, i.e., for small dip angles where the coaxial is least sensitive to these effects, the coplanar is most sensitive, and for large dip angles where the coaxial is most sensitive, the coplanar is least sensitive.

\section{Acknowledgments}

The authors thank Petrobras for the grant and the scholarship that support this work through the research project number 2017/00424-6.

\section{References}

Anderson, B., T. Barber, R. Bastia, J. B. Clauvaud, B. Coffin, M. Das, R. Hayden, T. Klimentos, C. C. Minh, and S. Williams, 2008, Triaxial induction - a new angle for an old measurement: Oilfield Review, 20, 64-84.

Anderson, B., T. Barber, and T. M. Habashy, 2002, The interpretation and inversion of fully triaxial induction data; a sensitivity study: 43rd SPWLA Annual Logging Symposium, SPWLA, Paper 0.

Anderson, B., S. Bonner, M. G. Lüling, and R. Rosthal, 1992, Response of 2-MHz LWD resistivity and wireline induction tools in dipping beds and laminated formations: The Log Analyst, 33, 461-475.

Carvalho, P. R. d., W. G. dos Santos, and C. Régis, 2010, Fundamentals of coaxial and coplanar coil arrays in induction tools: Brazilian Journal of Geophysics, 28, 1936.

Carvalho, P. R. d., C. R. T. Régis, and V. da Silva e Silva, 2018, Effects of the deviation angle of the borehole in the induction anisotropy logs: Brazilian Journal of Geophysics, 36, 291-399.

Clavaud, J.-B., R. Nelson, and U. K. Guru, 2005, Field example of enhanced hydrocarbon estimation in thinly laminated formation with a triaxial array induction tool: A laminated sand-shale analysis with anisotropic shale: SPWLA 46th Annual Logging Symposium, SPWLA, paper WW.

Gomes, R. M., P. S. Denicol, A. M. V. da Cunha, M. S. de Souza, B. F. Kriegshäuser, C. J. Payne, and A. Santos, 2002, Using multicomponent induction log data to enhance formation evaluation in deepwater reservoirs from campus basin, offshore brazil: 43rd SPWLA Annual Logging Symposium, Soc. Prof. Well Log. AnalystsSPWLA, Paper N.

Jin, J.-M., 2015, The finite element method in electromagnetics, third ed.: Wiley.

Kaufman, A. A., and G. Ytskovich, 2017, Basic principles of induction logging - Electromagnetic methods in borehole geophysics: Elsevier.

Key, K., 2012, Is the fast hankel transform faster than quadrature?: Geophysics, 77, F21-F30.

Kriegshäuser, B., O. Fanini, S. Forgang, G. Itskovich, M. Rabinovich, L. Tabarovsky, L. Yu, M. Epov, P. Gupta, and J. v d Horst, 2000, A new multicomponent induction logging tool to resolve anisotropic formations: Presented at the SPWLA 41st Annual Logging Symposium, Society of Petrophysicists and Well-Log Analysts.

Moinfar, A., C. Torres-Verdin, R. K. Mallan, and R. Angeles, 2010, Time-lapse variations of multi-component electrical resistivity measurements acquired in high-angle wells: Petrophysics, $\mathbf{5 1 .}$

Régis, C., P. R. de Carvalho, and V. da Silva e Silva, 2020, A new look at the causes of "polarization" horns in electromagnetic well logging: GEOPHYSICS, 85, D233D243.

Ward, S. H., and G. W. Hohmann, 1987, Electromagnetic theory for geophysical applications, in Nabighian, M. N., ed., Electromagnetic Methods in Applied Geophysics, Vol. 1, Theory: SEG, volume 1 of Investigations in Geophysics, 130-311.

Waxman, M., and L. Smits, 1968, Electrical Conductivities in Oil-Bearing Shaly Sands: Society of Petroleum Engineers Journal, 8, 107-122.

Zhang, Z., B. Yu, and C. Liu, 2012, Investigation of effects of large dielectric constants on triaxial induction logs: Applied Mathematics, 3, 1811-1817. 

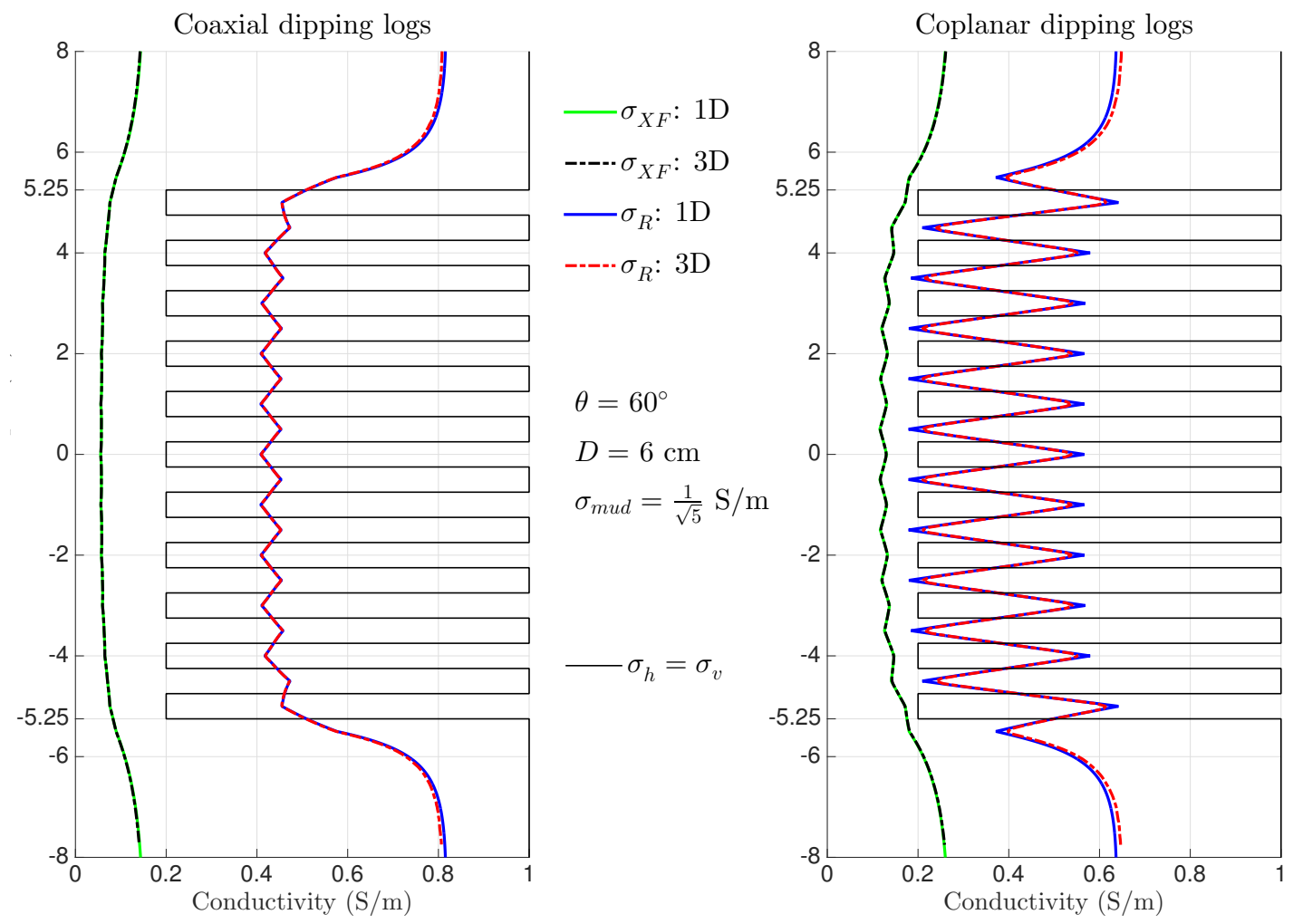

Figure 2: Validation test. Simulation of logs from a dipping well. 3D results generated with a thin borehole with a $6 \mathrm{~cm}$ diameter and mud conductivity equal to the geometric mean of the two conductivities in the model.
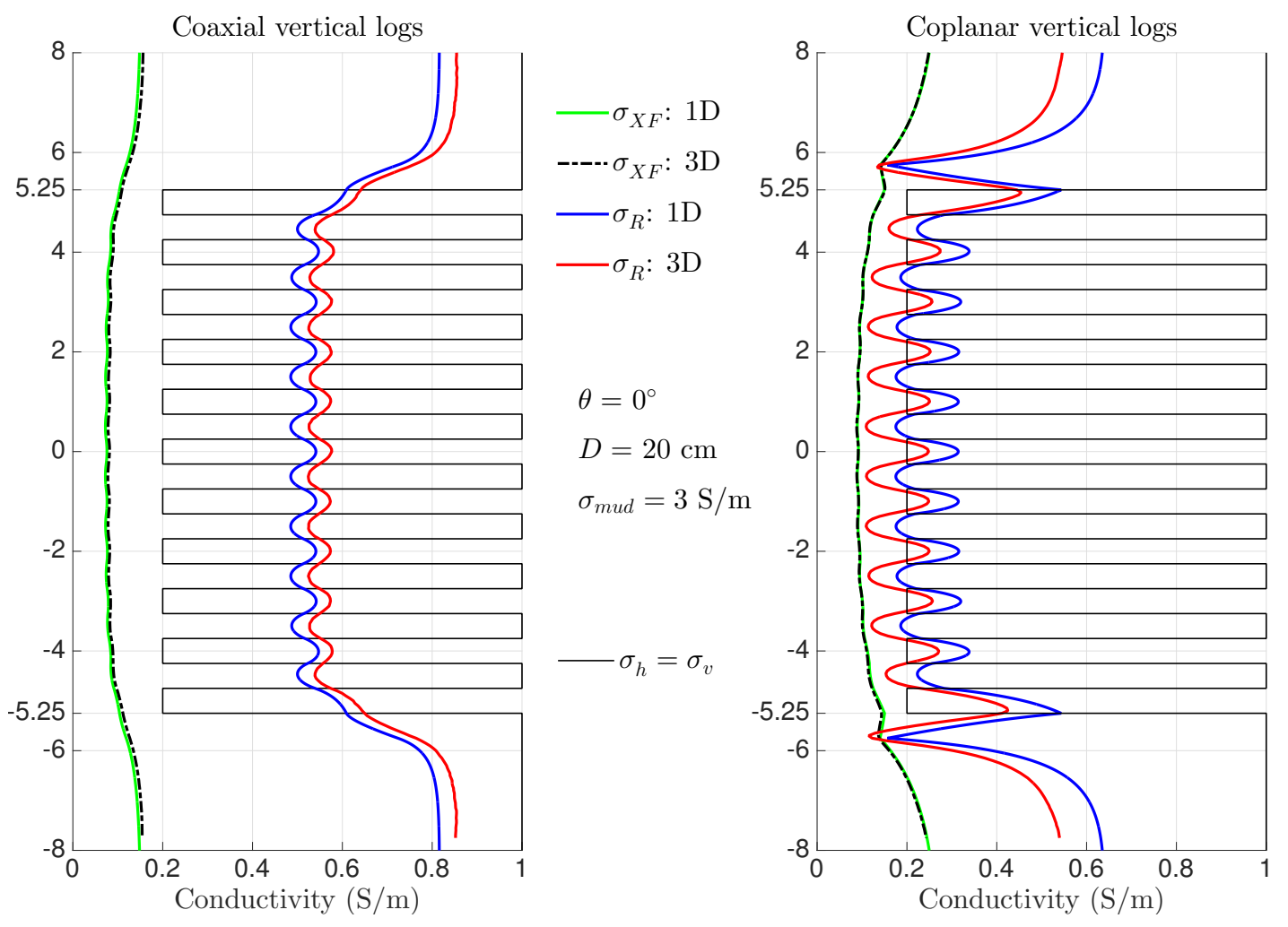

Figure 3: Comparison between logs without (1D) and with (3D) the influence of a borehole in a vertical well in an isotropic shale/sand laminated formation. 

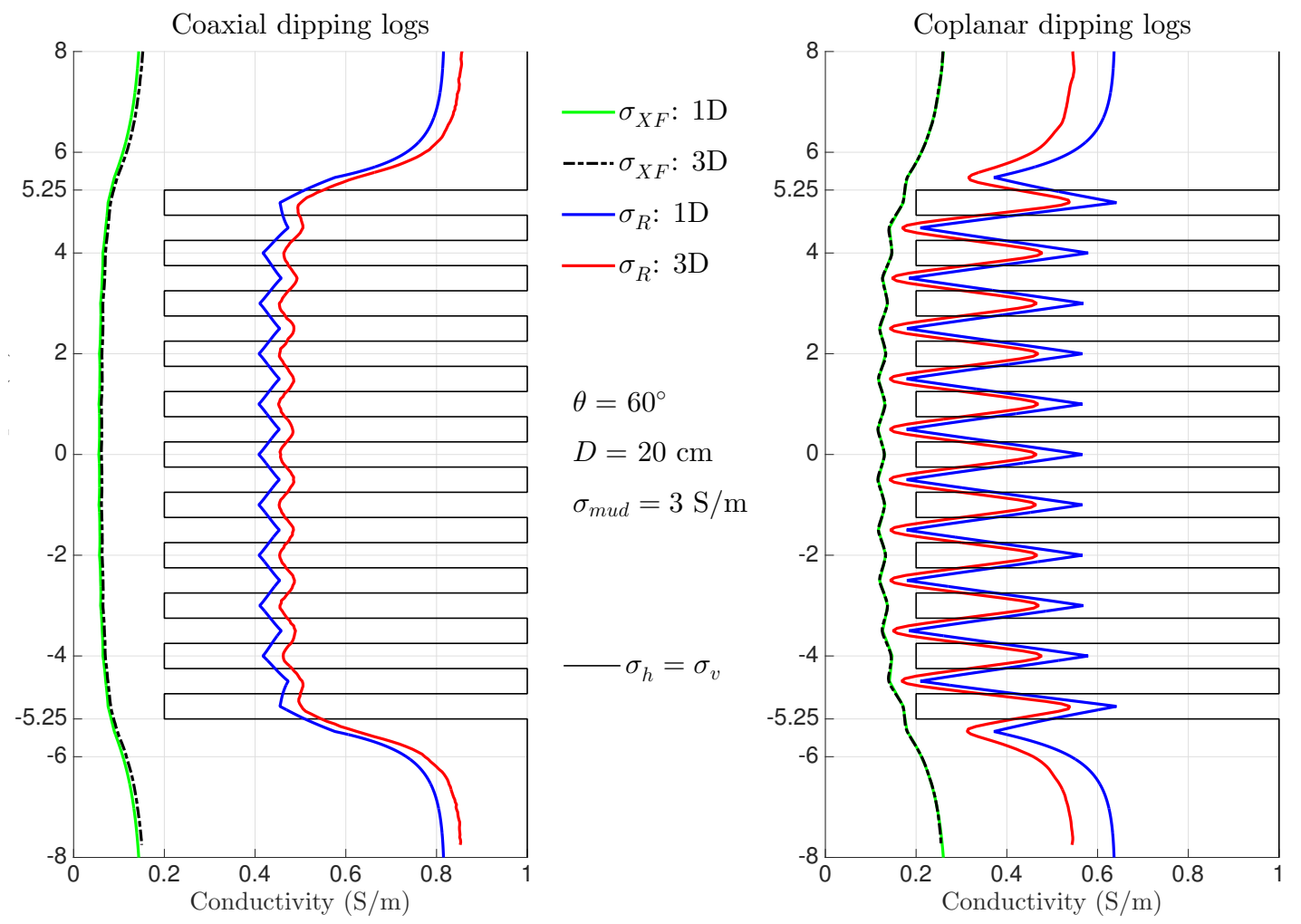

Figure 4: Comparison between logs without (1D) and with (3D) the influence of a borehole in a dipping well in an isotropic shale/sand laminated formation.
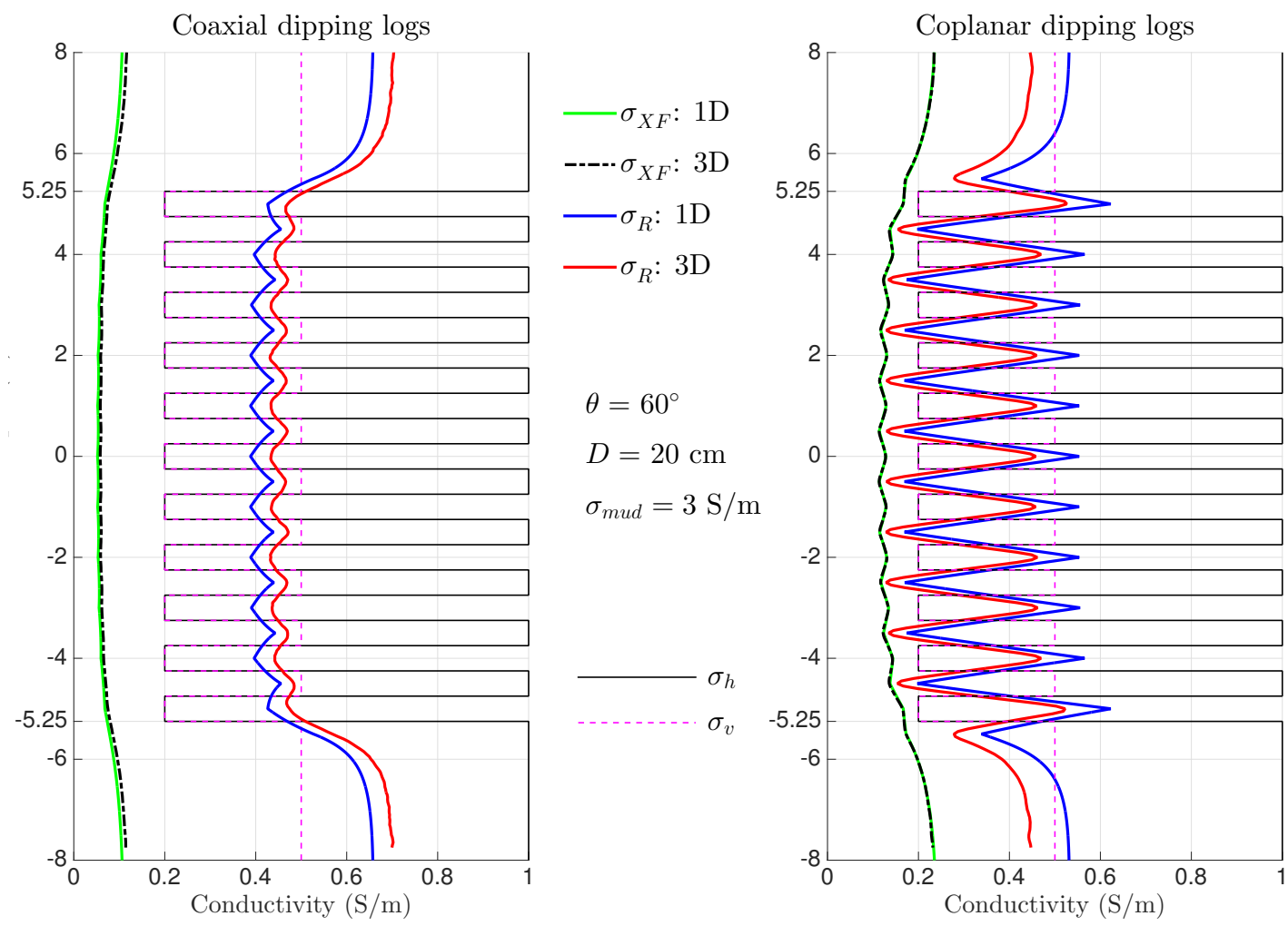

Figure 5: Comparison between logs without (1D) and with (3D) the influence of a borehole in a dipping well in a model composed of a sequence of isotropic sand laminae in an anisotropic shale host. 